\title{
AMENDMENTS
}

\section{Author Correction: Mechanosensing through immunoreceptors}

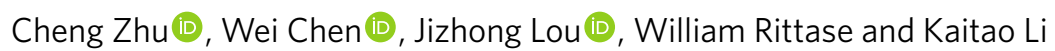

Correction to: Nature Immunology https://doi.org/10.1038/s41590-019-0491-1, published online 18 September 2019.

In the version of this article initially published, some citations of Fig. 3 in the second subsection ('Dissociation kinetics and conformational changes') were incorrect. The correct citations are as follows: first paragraph, seventh sentence, “...complexes ${ }^{15}$ (Fig. 3a)"; third paragraph, second sentence, “...the catch bond (Fig. 3a)...the MHC (Fig. 3b)"; third paragraph, third sentence, “...in the MHC ${ }^{19}$ that further..." (no citation); third paragraph, fifth sentence, “...changes (Fig. 3b)...slip bonds (Fig. 3a)"; third paragraph, sixth sentence, "... non-self (Fig. 3b)"; fourth paragraph, second sentence, “...coupling (Fig. 3c)”; fourth paragraph, third sentence, “...HLA-B44 (Fig. 3d)...”. The errors have been corrected in the HTML and PDF versions of the article.

Published online: 4 November 2019

https://doi.org/10.1038/s41590-019-0545-4

(c) Springer Nature America, Inc. 2019

\section{Publisher Correction: Innate-like functions of natural killer T cell subsets result from highly divergent gene programs}

Isaac Engel, Grégory Seumois, Lukas Chavez, Daniela Samaniego-Castruita, Brandie White, Ashu Chawla, Dennis Mock, Pandurangan Vijayanand and Mitchell Kronenberg

Correction to: Nature Immunology https://www.nature.com/articles/ni.3437, published online 18 April 2016.

In the version of this article initially published, the labels above the third and fourth groups in Fig. 2b were switched; the far right label (above the fourth, pink group) should be NKT17, and the label just to its left (above the third, green group) should be NKT1. Also, the third gene symbol (Ccn $2 b)$ along the right margin of Fig. 3a was incorrect; the correct gene symbol is Ccnb2. Finally, the gene symbols $(C 1 k d$ and $C c n 2 b)$ above the far left plots in Fig. 3b were incorrect; the correct gene symbols are Cdk1 (top left plot) and Ccnb2 (bottom left plot). 